\title{
Serum homocysteine levels are decreased in levothyroxine-treated women with autoimmune thyroiditis
}

Maciej Owecki ${ }^{*}$, Jolanta Dorszewska ${ }^{2}$, Nadia Sawicka-Gutaj ${ }^{1}$, Anna Oczkowska ${ }^{2}$, Michał K Owecki ${ }^{2}$, Michał Michalak ${ }^{3}$, Jakub Fischbach', Wojciech Kozubski ${ }^{1}$ and Marek Ruchała'

\begin{abstract}
Background: Hyperhomocysteinemia is a well-known cardiovascular risk factor and its elevation is established in overt hypothyroidism. Since some authors suggest that chronic autoimmune thyroiditis per se may be considered as a novel risk factor of atherosclerosis independent of thyroid function, the analysis of classical cardiovascular risk factors might be helpful in evaluation the causative relationship. Data concerning the impact of thyroid autoimmunity in euthyroid state on homocysteine (Hcy) level is lacking. The aim of this study was to evaluate Hcy level in context of anti-thyroperoxidase antibodies (TPOAbs) in euthyroidism.
\end{abstract}

Methods: It is a case-control study. 31 euthyroid women treated with levothyroxine (L-T4) due to Hashimoto thyroiditis (HT) and 26 females in euthyroidism without L-T4 replacement therapy were enrolled in the study. All women with HT had positive TPOAbs. Forty healthy females negative for TPOAbs comparable for age and body mass index (BMI) participated in the study as controls. Exclusion criteria were a history of any acute or chronic disease, use of any medications (including oral contraceptives and vitamin supplements), smoking, alcoholism.

Results: TPOAbs titers were higher in both groups of HT patients versus the healthy controls. Hcy levels were found to be significantly lower in treated HT patients (Me $11 \mu \mathrm{mol}$; IQR $4.2 \mu \mathrm{mol}$ ) as compared with healthy controls (Me 13.35 umol; IQR 6.34 umol; p = 0.0179). In contrast, no significant difference was found between non treated HT and control group in Hcy level. The study groups and the controls did not differ in age and BMI. Furthermore, levels of TSH, FT4, TC, LDL, HDL and TAG did not differ between the study group and the control group.

Conclusion: The main finding of the study is a decrease in Hcy level in treated HT as compared with healthy controls. Based on our observations one can also assume that correct L-T4 replacement was associated here with a decrease of Hcy. Furthermore, it seems that non treated HT in euthyroidism is not associated with Hcy increase, in contrast to overt hypothyroidism. This may be just another argument against the concepts about the role of "euthyroid HT" in the development of atherosclerosis.

Keywords: Homocysteine, Thyroid, Autoimmunity, Hashimoto disease

\footnotetext{
*Correspondence: mowecki@ump.edu.pl

'Department of Endocrinology, Metabolism and Internal Medicine, Poznan University of Medical Sciences, Przybyszewskiego St. 49, 60-355 Poznań, Poland

Full list of author information is available at the end of the article
} 


\section{Background}

Homocysteine (Hcy) is a sulfur-containing amino acid naturally found in human blood and its metabolism is based on two divergent pathways: transsulfuration and remethylation [1]. Hcy has been investigated as a risk factor for cardiovascular disease since 1969, when McCully observed that two patients with homocystinuria were affected with extensive atherosclerosis and arterial thrombosis [2]. Since the association between elevated level of Hcy and increased risk of coronary heart disease was established $[3,4]$, the issue whether there is a causal relation still remains unclear $[5,6]$.

To date, hypothyroidism is considered as an independent risk factor for atherosclerosis. However, the atherogenic lipid profile does not fully explain the increased cardiovascular morbidity in hypothyroid individuals. For that reason, the possible association between hypothyroid state and Hcy concentration was suggested. Interestingly, increased Hcy level in overt hypothyroidsm was found in many studies [7-9]. Additionally, normalization of Hcy level was achieved after euthyroidism restoration [10]. In sharp contrast with the above mentioned results, decreased Hcy concentration was found in hyperthyroidism [11].

The possible mechanism responsible for increased Hcy level in hypothyroidism also remains a matter of recent debate. Firstly, the observed hyperhomocysteinemia may reflect impaired renal Hcy clearance. Hypothyroidism probably reduces glomerular filtration rate leading to increased creatinine and Hcy levels [12-14]. Secondly, impaired liver metabolism of Hcy linked with hypothyroidism may contribute to hyperhomocysteinemia. Decreased activity of both enzymes, methionine synthase and methylenetetrahydrofolate reductase was established in thyroidectomized rats and may also explain the elevated level of Hcy in hypothyroidism [15-17].
In contrast to overt thyroid disorders, data concerning Hcy concentration among patients with subclinical hypothyroidism $(\mathrm{SH})$ is contradictory. Some studies showed that, despite atherogenic lipid profile in $\mathrm{SH}$, Hcy level is not increased $[18,19]$. On the other hand, Hcy concentration was reported to be higher as compared with euthyroid controls [20].

In view of those controversies, and considering the fact that numerous thyroid disorders are caused by autoimmune disturbances, we hypothesized that it might be the autoimmunity against thyroid gland that initially affects Hcy production, even in pre-clinical phases of thyroid disorders. Therefore, the aim of this study was to evaluate Hcy level in context of anti-thyroperoxidase antibodies (TPOAbs) rather than thyroid function. To achieve this goal, Hcy concentration was determined in otherwise healthy and euthyroid women with, and without chronic autoimmune thyroiditis. The criterion of euthyroidism let us exclude the possible influence of thyroid dysfunction per se on Hcy concentrations.

\section{Methods}

Thirty one euthyroid women treated with levothyroxine (L-T4) due to Hashimoto thyroiditis (HT) at the outpatient clinic of the Department of Endocrinology, Metabolism and Internal Medicine and twenty six females with chronic autoimmune thyroiditis in euthyroidism without L-T4 replacement therapy were enrolled in the study (Table 1). All women with HT had positive TPOAbs. Forty healthy females negative for TPOAbs comparable for age and body mass index (BMI) participated in the study as controls (Table 1 ).

All subjects and controls were euthyroid, either spontaneously, or under L-T4 medication. None of the patients and the controls had a history of any acute or chronic disease, including diabetes mellitus, hypertension, angina

Table 1 Characteristics of the study groups and the controls

\begin{tabular}{|c|c|c|c|c|}
\hline & Non treated Hashimoto $(n=26)$ & Treated Hashimoto $(n=31)$ & Controls $(n=40)$ & $\mathrm{p}$ \\
\hline Age (yr) & $43(17)$ & $38(18)$ & $35.5(15)$ & ns \\
\hline $\mathrm{BMI}\left(\mathrm{kg} / \mathrm{m}^{2}\right)$ & $22.8(3.6)$ & $22.6(4)$ & $21.8(5.40)$ & ns \\
\hline $\mathrm{TSH}(\mathrm{mU} / \mathrm{L})$ & $1.64(2.08)$ & $2.07(3.14)$ & $1.54(1.5)$ & ns \\
\hline FT4 (pmol/L) & $14.7(2.27)^{\mathrm{a}}$ & $17.13(5.11)^{b}$ & $15.52(2.23)^{a, b}$ & $p=0.0019$ \\
\hline TPOAbs (IU/mL) & $242(290)^{b}$ & $300(391)^{b}$ & $9(5.5)^{\mathrm{a}}$ & $p<0.0001$ \\
\hline Hcy ( $\mu \mathrm{mol})$ & $11(4.2)^{a, b}$ & $10.8(6.9)^{\mathrm{a}}$ & $13.35(6.34)^{b}$ & $p=0.0179$ \\
\hline$[$ Mean \pm SD $]$ & {$[10.33 \pm 3.36]^{a, b}$} & {$[9.84 \pm 4.24]^{a}$} & {$[12.97 \pm 6.71]^{b}$} & \\
\hline $\mathrm{TC}(\mathrm{mg} / \mathrm{dl})$ & $207(64)$ & $205(60)$ & $196(51)$ & ns \\
\hline $\mathrm{LDL}(\mathrm{mg} / \mathrm{dl})$ & 118.5 (50.6) & $121(45.6)$ & 110.9 (37.4) & ns \\
\hline $\mathrm{HDL}(\mathrm{mg} / \mathrm{dl})$ & $57(30)$ & $67(16)$ & 65 (15.5) & ns \\
\hline TAG (mg/dl) & 75 (38) & $83(47)$ & $82(46)$ & ns \\
\hline
\end{tabular}

Results are expressed as medians, followed by interquartile ranges given in brackets. $a, b-$ values followed by the same letters do not differ significantly at $p<0.05$. 
pectoris, evidence of any kidney or liver disorder. Exclusion criteria were also use of any medications (including oral contraceptives and vitamin supplements), smoking, alcoholism.

All subjects underwent physical examination with recording of height, weight, heart rate, systolic and diastolic blood pressure. Blood samples were obtained after an overnight fast. In patients with HT blood samples were drawn before the ingestion of usual morning L-T4 medication. Serum levels of thyrotropin hormone (TSH), free thyroxine (FT4), TPOAbs, Hcy, total cholesterol (TC), low-density lipoprotein (LDL), high-density lipoprotein (HDL), triacylglycerol (TAG) were evaluated.

TSH and FT4 were measured using the electrochemiluminescence technique in Cobas E 601 (norm ranges: TSH 0.27-4.2 mU/l; FT4 11.5-21.0 pmol/l). TPOAbs were measured by radioimmunoassay (norm range: $<34 \mathrm{IU} / \mathrm{ml}$ ).

Serum Hcy levels were assessed by high-performance liquid chromatography (HPLC). The analyzed plasma thiol compounds (Hcy, Fluka Germany) were diluted with water at 2:1 ratio and reduced using 1\% TCEP (Tris-(2-carboxyethyl)-phosphin-hydrochloride; Applichem, Germany) at 1:9 ratio. Subsequently, the sample was deproteinized using $1 \mathrm{M} \mathrm{HClO}_{4}$ (at 2:1 ratio) and applied to the HPLC/EC system.

To determine thiol concentration the samples were fed to the HPLC system (P580A; Dionex, Germany) coupled to an electrochemical detector (CoulArray 5600; ESA, USA). The analysis was performed in Termo Hypersil BDS C18 column $(250 \mathrm{~mm} \times 4.6 \mathrm{~mm} \times 5 \mu \mathrm{m})$ (Germany) in isocratic conditions, using the mobile phase of $0.15 \mathrm{M}$ phosphate buffer, $\mathrm{pH}$ 2.9, supplemented with $12.5-17 \%$ acetonitrile. The system was controlled, and the data were collected and processed using Chromeleon software (Dionex, Germany).

The study was approved by the Poznan University of Medical Sciences Ethical Commitee, and informed consent was signed by every subject.

Comparison of analyzed parameters between three groups (HT patients versus healthy controls) was performed by Kruskal-Wallis test with Dunn's post-hoc tests because data did not follow normal distribution. Normality was analyzed by Shapiro-Wilk's test. Results were presented as medians and interquartile ranges (IQR). Spearman's correlation coefficient was used to measure the strength of relationship of analyzed parameters. All tests were performed two-tailed and were considered as significant at $\mathrm{p}<0.05$. Statistical analyses were performed with Statistica 10 (StatSoft Inc., Poland) and MedCalc version 10.3.2 (MedCalc Software, Mariakerke, Belgium) .

\section{Results}

Descriptive characteristics for the study groups and the control group are shown in Table 1.
The study groups and the controls did not differ in age and BMI.

TSH levels of treated and non treated HT patients were similar to those of control subjects. FT4 concentrations of treated HT patients were significantly higher as compared with non treated HT and did not differ from controls (Figure 1). TPOAbs titers were higher in both groups of HT patients versus the healthy controls.

Hcy levels were found to be significantly lower in treated HT patients as compared with healthy controls (Figure 2). In contrast, no significant difference was found between non treated HT and control group in Hcy level.

In treated HT females Hcy level was negatively correlated with age $(\mathrm{p}=0.017289 ; \mathrm{r}=-0.42)$. In contrast, correlation between Hcy and age was not found in non treated HT and in controls. No significant associations were found between Hcy and BMI, waist circumference, TSH, FT4, TPOAbs in all of HT patients and in healthy females. Furthermore, levels of TC, LDL, HDL and TAG did not differ between the study groups and the control group.

\section{Discussion}

In this study, we investigated the influence of thyroid autoimmunity on Hcy concentrations. To achieve this goal, we used very strict criteria of enrollment, and we excluded the possible influence of hypothyroidism by including only patients who were euthyroid, obviously either spontaneously, or under medication. With this novel approach, we came to somewhat surprising conclusions that we discuss beneath.

We showed here that non treated HT and treated HT females had comparable TPOAbs, however treated HT group had higher level of FT4. Moreover, FT4 in treated HT and healthy controls did not differ indicating sufficient replacement therapy. The main finding of the study is a decrease in Hcy level in treated HT as compared with healthy controls.

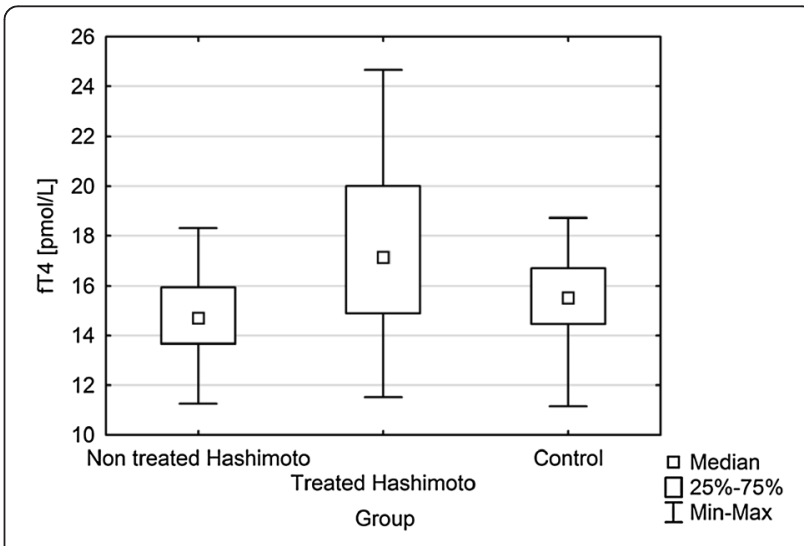

Figure 1 FT4 concentrations in non treated and treated HT and in controls. 




Figure 2 Hcy levels in non treated and treated HT and in controls.

Obviously, our findings should be understood in a broader context of the association between thyroid autoimmunity and atherosclerosis. Indeed, some authors suggest that chronic autoimmune thyroiditis per se may be considered as a novel risk factor of atherosclerosis independent of thyroid function [21-24]. Currently, however, the causative relationship between thyroid autoimmunity and increased risk of atherosclerosis has not yet been established. A few studies which were addressing this question investigated the effect of thyroid autoimmunity on lipid profile, abdominal obesity, fasting glucose and homeostasis model assessment (HOMA) insulin resistance, as well as carotid intima-media thickness (CIMT) [25-27]. However, in contrast to our study group, the study populations were not uniform, therefore the conclusions of these investigations are not comparable. Tamer et al. found that euthyroid HT $(\mathrm{n}=84)$ patients had higher LDL level as compared with controls $(\mathrm{n}=$ 150) $(\mathrm{p}=0.0042)$ [25]. Moreover, TPOAbs level was negatively correlated with HDL $(\mathrm{p}=0.031 ; \mathrm{r}=-0.137)$ and positively with TAG $(\mathrm{p}=0.013 ; \mathrm{r}=0.158)$ and waist circumference $(p=0.048 ; r=0.128)$. Ciccone et al. established that overweight or obese women with HT have increased IMT as compared with controls [27]. They suggested that the autoimmunity in HT patients is an independent factor that might accelerate atherosclerosis. However, they also found that HT patients had higher TSH levels and lower FT3.

It must be emphasized here that the effect of both hypothyroidism and hyperthyroidism on Hcy concentration has been investigated in many studies before. However, data concerning the impact of thyroid autoimmunity in euthyroid state on Hcy level is lacking.

To our knowledge, there was only one report of Hcy in context of thyroid autoimmunity in euthyroid premenopausal females with Hashimoto thyroiditis recently reported by Topaloglu et al. [26]. In this research, the study population was divided into two subgroups: first with $\mathrm{TSH} \leq 2.5 \mathrm{IU} / \mathrm{L}$ and second with $\mathrm{TSH}>2.5 \mathrm{IU} / \mathrm{L}$. The controls were age-matched. CIMT was the only one evaluated parameter that was significantly higher in the study group regardless of TSH. CIMT was positively correlated with antithyroglobulin antibodies $(\mathrm{p}=0.014$; $r=0.328$ ). In contrast to our results, Hcy level did not differ between the study and the control groups. However, in our study the control group had comparable BMI with the study group, therefore the body weight did not have any effect on the Hcy comparison. In contrast, Topaloglu et al. had a control group with BMI much lower than the study group $(\mathrm{p}<0.001)$. Therefore, the potential influence of this co-variable on the Hcy analysis should be taken into consideration. New insight into the discussed problem was given in recently published research concerning Hcy level in patients with atrophic glossitis or burning mouth syndrome and autoimmune thyroiditis [28]. Wang et al. found increased Hcy level in these patients independently of thyroid function. Majority of the studied group positive for antithyroid antibodies (anti-thyroglobulin antibodies or antithyroid microsomal antibodies) was euthyroid. However, despite normal thyroid function in $85.8 \%$ of investigated individuals abnormal high blood Hcy level was established. Authors suggested that this finding was linked with vitamin B12 deficiency confirmed among this group.

Our study has some strengths and limitations. The main limitation of the present study is the number of patients who underwent the evaluation. However, it should be underlined, that all subjects were euthyroid females without any comorbidities and they did not take any medication. The only one difference between treated HT females and controls were TPOAbs. In our opinion, the homogeneity of subjects examined was the strength of this work that could balance its limitations.

Serum FT4 concentration is considered as an independent determinant of Hcy level [29]. As was mentioned above, Hcy level is generally decreased in hyperthyroid patients in contrast to hypothyroid subjects, who have higher Hcy concentration. Moreover, restoration of thyroid function leads to normalization of Hcy concentration. In general, our study and control groups were euthyroid, but this state was achieved by L-T4 replacement therapy in a group of treated HT women. This group had lower Hcy levels than normal controls, despite similar FT4 levels. A possible explanation of this finding is the fact that, in spite of similar hormone concentrations, these patients differed in that had different sources of thyroxine: it was endogenous in one group, and exogenous in the other. In our opinion, there is a causative relationship between L-T4 replacement therapy and decreased Hcy levels. Patients who are on L-T4 therapy 
have daily changes of FT4 serum concentration that result from pharmacokinetic properties of this medication. The maximum FT4 concentration occurs approximately two hours after the drug ingestion [30]. Moreover, there is a transient increase of FT4 serum level after ingestion of L-T4 for 5 hours [31]. Since FT4 directly influences Hcy concentration, during this time Hcy metabolism is similar to hyperthyroid state and it may lead to decreased Hcy levels in treated HT patients in contrast to healthy controls, in whom FT4 output is adjusted to real needs and the rate of physiological elimination .

\section{Conclusions}

The main finding of the study is a decrease in Hcy level in treated HT as compared with healthy controls. Our study adds further evidence to the debate on possible association between chronic autoimmune thyroiditis and atherosclerosis. It seems that non treated HT in euthyroidism is not associated with Hcy increase, in contrast to overt hypothyroidism. This may be just another argument against the concepts about the role of "euthyroid HT" in the development of atherosclerosis, which is of quite importance considering the high prevalence of high TPOAbs titers in Europe. Furthermore, that Hcy was lower in the treated group may point to the beneficial role of L-T4 treatment in general. Correct L-T4 replacement was associated here with a decrease of Hcy. In conclusion, L-T4 treatment may further add to medical approach aimed at atherosclerosis risk reduction with regard to Hcy decrease in L-T4-treated women with chronic autoimmune thyroiditis. This last concept, however, as drawn only from a cross-sectional setting, requires further investigation in observational studies.

\section{Competing interests}

The authors declare that there are no conflicts of interests.

\section{Authors' contributions}

MO conceived of the study, and participated in its design and coordination and recruitment of patients, drafted the manuscript; JD participated in design of the study, carried out the high-performance liquid chromatography and helped to draft the manuscript; NSG collected and analyzed the data, prepared the manuscript, performed the statistical analysis and the literature review; AO carried out the immunoassays and high-performance liquid chromatography; MKO participated in design of the study and coordination; MM performed the statistical analysis, helped to draft the manuscript; JF participated in design of the study and recruitment of patients; WK corrected the manuscript, approved the final version of the manuscript; MR corrected the manuscript, approved the final version of the manuscript. All authors read and approved the final manuscript.

\section{Funding}

The total cost of measurements (immunoassays and high-performance liquid chromatography techniques) were funded by Poznan University of Medical Sciences Internal Funding. The funder had no role in study design, data collection and analysis, decision to publish, or preparation of manuscript. The authors have not been paid to carry out the study and write the manuscript.

\section{Author details}

'Department of Endocrinology, Metabolism and Internal Medicine, Poznan University of Medical Sciences, Przybyszewskiego St. 49, 60-355 Poznań, Poland. ${ }^{2}$ Department of Neurology, Poznan University of Medical Sciences, Przybyszewskiego St. 49, 60-355 Poznań, Poland. ${ }^{3}$ Department of Informatics and Statistics, Poznan University of Medical Sciences, Dąbrowskiego St. 79, 60-529 Poznań, Poland.

Received: 25 November 2013 Accepted: 25 February 2014 Published: 1 March 2014

\section{References}

1. Selhub J: Homocysteine metabolism. Annu Rev Nutr 1999, 19:217-246.

2. McCully KS: Vascular pathology of homocysteinemia: implications for the pathogenesis of arteriosclerosis. Am J Pathol 1969, 56:111-128.

3. Homocysteine Studies Collaboration: Homocysteine and risk of ischemic heart disease and stroke: a meta-analysis. JAMA 2002, 288:2015-2022.

4. Klerk M, Verhoef P, Clarke R, Blom HJ, Kok FJ, Schouten EG: MTHFR Studies Collaboration Group MTHFR $677 \mathrm{C} \rightarrow$ T polymorphism and risk of coronary heart disease: a meta-analysis. JAMA 2002, 288:2023-2032.

5. Thampi P, Stewart BW, Joseph L, Melnyk SB, Hennings $\amalg$, Nagarajan S: Dietary homocysteine promotes atherosclerosis in apoE-deficient mice by inducing scavenger receptors expression. Atherosclerosis 2008, 197:620-629.

6. Clarke R, Halsey J, Bennett D, Lewington S: Homocysteine and vascular disease: review of published results of the homocysteine-lowering trials. J Inherit Metab Dis 2011, 34:83-91.

7. Nedrebø BG, Ericsson UB, Nygård O, Refsum H, Ueland PM, Aakvaag A, Aanderud S, Lien EA: Plasma total homocysteine levels in hyperthyroid and hypothyroid patients. Metabolism 1998, 47:89-93.

8. Morris MS, Bostom AG, Jacques PF, Selhub J, Rosenberg $\mathrm{HH}$ : Hyperhomocysteinemia and hypercholesterolemia associated with hypothyroidism in the third US National Health and Nutrition Examination Survey. Atherosclerosis 2001, 155:195-200.

9. Gunduz M, Gunduz E, Kircelli F, Okur N, Ozkaya M: Role of surrogate markers of atherosclerosis in clinical and subclinical thyroidism. Int J Endocrinol 2012. doi:10.1155/2012/109797.

10. Lien EA, Nedrebø BG, Varhaugh JE, Nygard O, Aakvaag A, Ueland PM: Plasma total homocysteine levels during short-term iatrogenic hypothyroidism. J Clin Endocrinol Metab 2000, 85:1049-1053.

11. Nedrebø BG, Nygård O, Ueland PM, Lien EA: Plasma total homocysteine in hyper- and hypothyroid patients before and during 12 months of treatment. Clin Chem 2001, 47:1738-1741.

12. Diekman MJ, van der Put NM, Blom HJ, Tijssen JG, Wiersinga WM: Determinants of changes in plasma homocysteine in hyperthyroidism and hypothyroidism. Clin Endocrinol (Oxf) 2001, 54:197-204.

13. Lien EA, Nedrebø BG, Varhaug JE, Nygård O, Aakvaag A, Ueland PM: Plasma tHcy levels during short-term iatrogenic hypothyroidism. J Clin Endocrinol Metab 2000, 85:1049-1053.

14. Nedrebø BG, Nygård O, Ueland PM, Lien EA: Plasma tHcy in hyper- and hypothyroid patients before and during 12 months of treatment. Clin Chem 2001, 47:1738-1741.

15. Chan MM, Stokstad EL: Metabolic responses of folic acid and related compounds to thyroxine in rats. Biochimica Biophysica Acta 1980, 632:244-253.

16. Nair CP, Viswanathan G, Noronha JM: Folate-mediated incorporation of ring-2-carbon of histidine into nucleic acids: influence of thyroid hormone. Metabolism 1994, 43:1575-1578.

17. Ayav A, Alberto JM, Barbe F, Brunaud L, Gerard P, Merten M, Gueant JL: Defective remethylation of homocysteine is related to decreased synthesis of coenzymes B2 in thyroidectomized rats. Amino Acids 2005, 28:37-43.

18. Luboshitzky R, Aviv A, Herer P, Lavie L: Risk factors for cardiovascular disease in women with subclinical hypothyroidism. Thyroid 2002, 12:421-425.

19. Atabek ME, Pirgon O, Erkul I: Plasma homocysteine concentration in adolescents with subclinical hypothyroidism. J Pediatr Endocrinol Metab 2003, 16:1245-1248.

20. Sengul E, Cetinarslan B, Tarkun I, Canturk Z, Turemen E: Homocysteine concentrations in subclinical hypothyroidism. Endocr Res 2004, 30:351-359. 
21. Bastenie PA, Vanhaelst L, Golstein J, Smets P: Asymptomatic autoimmune thyroiditis and coronary heart disease.Cross-sectional and prospective studies. Lancet 1977, 2:155-158.

22. Hak AE, Pols HA, Visser TJ, Drexhage HA, Hofman A, Witteman JC: Subclinical hypothyroidism is an independent risk factor for atherosclerosis and myocardial infarction in elderly women: the Rotterdam study. Ann Intern Med 2000, 132:270-278.

23. Nyirenda MJ, Clark DN, Finlayson AR, Read J, Elders A, Bain M, Fox KA, Toft AD: Thyroid disease and increased cardiovascular risk. Thyroid 2005, 15:718-724.

24. Zoller B, Li X, Sundquist J, Sundquist K: Risk of subsequent ischemic and hemorrhagic stroke in patients hospitalized for immune-mediated diseases: a nationwide follow-up study from Sweden. BMC Neurol 2012, $12: 41$.

25. Tamer G, Mert M, Tamer I, Mesci B, Kilic D, Arik S: Effects of thyroid autoimmunity on abdominal obesity and hyperlipideamia. Endokrynol Pol 2011, 62:421-428

26. Topaloglu O, Gokay F, Kucukler K, Burnik FS, Mete T, Yavuz HC, Berker D, Guler S: Is autoimmune thyroiditis a risk factor for early atherosclerosis in premenopausal women even if in euthyroid status? Endocrine 2013, 44:145-151.

27. Ciccone MM, De Pergola G, Porcelli MT, Scicchitano P, Caldarola P, Lacoviello M, Pietro G, Giorgino F, Favale S: Increased carotid IMT in overweight and obese women affected by Hashimoto's thyroiditis: an adiposity and autoimmune linkage? BMC Cardiovasc Disord 2010, 10:22.

28. Wang YP, Lin HP, Chen HM, Kuo YS, Lang MJ, Sun A: Hemoglobin, iron, and vitamin B12 deficiencies and high blood homocysteine levels in patients with anti-thyroid autoantibodies. J Formos Med Assoc 2012. doi:10.1016/j.jfma.2012.04.003.

29. Orzechowska-Pawilojc A, Sworczak K, Lewczuk A, Babinska A: Homocysteine, folate and cobalamin levels in hypothyroid women before and after treatment. Endocr J 2007, 54:471-476.

30. Colucci P, Seng Yue C, Ducharme M, Benvenga S: A review of the pharmacokinetics of levothyroxine for the treatment of hypothyroidism. Eur Endocrinol 2013, 9:40-47.

31. Ain KB, Pucino F, Shiver TM, Banks SM: Thyroid hormone levels affected by time of blood sampling in thyroxine-treated patients. Thyroid 1993, 3:81-85.

doi:10.1186/1472-6823-14-18

Cite this article as: Owecki et al:: Serum homocysteine levels are decreased in levothyroxine-treated women with autoimmune thyroiditis. BMC Endocrine Disorders 2014 14:18.

\section{Submit your next manuscript to BioMed Central and take full advantage of:}

- Convenient online submission

- Thorough peer review

- No space constraints or color figure charges

- Immediate publication on acceptance

- Inclusion in PubMed, CAS, Scopus and Google Scholar

- Research which is freely available for redistribution 